\title{
Screening of Myxobacteria Carrying Tubulysin Biosynthetic Genes
}

\author{
Hyesook Hyun ${ }^{1}$, Juo Choi ${ }^{1,2}$, Daun Kang ${ }^{2}$, Yungpil Kim², Pilgoo Lee², Gregory J. Y. Chung ${ }^{2}$, and Kyungyun Cho ${ }^{1 *}$ \\ ${ }^{1}$ Department of Biotechnology, Hoseo University, Asan 31499, Republic of Korea \\ ${ }^{2}$ MECOX CureMed Co., Seoul 06744, Republic of Korea
}

Received: October 6, 2020 / Revised: November 5, 2020 / Accepted: November 28, 2020

Tubulysins are a group of secondary metabolites produced by myxobacteria that inhibit the function of the eukayotic cytoskeleton. We developed a pair of PCR primers that specifically amplified tubulysin biosynthetic genes. Using these primers, eight out of the eighty-one strains of myxobacteria belonging to the Cystobacteraceae family that harbored putative tubulysin biosynthetic genes were screened through PCR analysis. The selected strains included two Archangium gephyra, two Stigmatella sp., two Vitiosangium cumulatum, and two unidentified myxobacteria. LC-MS analysis of the culture extracts from the selected strains revealed that $A$. gephyra KYC4066 produced putative tubulysin A and B.

Keywords: Archangium gephyra, myxobacteria, secondary metabolite, tubulysin

\section{Introduction}

Myxobacteria are delta-proteobacteria that produce diverse bioactive secondary metabolites. More than 600 different metabolites have been isolated from myxobacteria [1-3]. Among these metabolites isolated from myxobacteria, seven groups of metabolites exhibit inhibitory activity against the function of the cytoskeleton in eukayotic cells. Substances inhibiting the function of the cytoskeleton possess the potential to be developed as anticancer drugs. For example, epothilones are a group of the substances produced by myxobacteria that inhibit cytoskeleton function [4]. The United States Food and Drug Administration approved Ixabepilone, a semisynthetic derivative of epothilone $\mathrm{B}$, as an anticancer treatment in 2007, as it exhibits excellent effects on cancer cells that possess multi-pharmaceutical resistance [5].

Tubulysins are another group of substances that inhibit the function of the cytoskeleton and have been

\section{*Corresponding author}

Tel: +82-41-540-5627, Fax: +82-41-548-6231

E-mail: kycho@hoseo.edu studied for its superior anticancer activity [6-9]. Tubulysins promote the depolymerization of tubulin polymers [10], while epothilones promote the polymerization of tubulin [4]. Tubulysins are attractive lead structures for development as antineoplastic agents; however, tubulysin-producing strains have not been isolated and reported in Korea.

Tubulysins were previously reported to be produced by the myxobacteria Angiococcus disciformis, Archangium gephyra, and Cystobacter sp. [6, 11]. Tubulysin biosynthetic gene clusters have been identified and reported from $A$. disciformis An d48 and Cystobacter sp. SBCb004 [11, 12] (Fig. 1). DNA sequences homologous to those of the tubulysin biosynthetic genes are also found in the genome of Vitiosangium sp. GDMCC 1.1324 (GenBank accession number: NZ_PZOX01000000). Angiococcus, Archangium, Cystobacter, and Vitiosangium genera belong to the Cystobacteraceae family of myxobacteria [13]. Myxobacteria belonging to Cystobacteraceae are distingushed from other myxobacterial families in the shape of vegetative cells, myxospores, and swarm colonies. Vegetative cells of Cystobacteraceae are slender, 


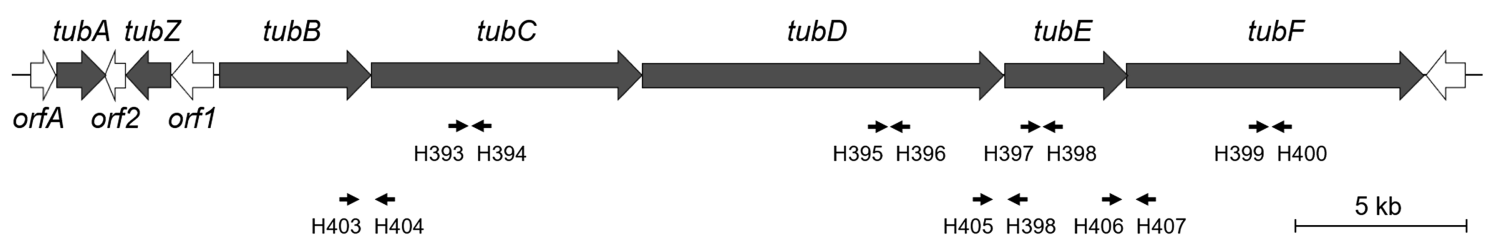

Fig. 1. PCR primers used to detect tubulysin biosynthetic genes. Locations of the primers are marked on the tubulysin biosynthetic gene cluster from Cystobacter sp. SBCb004 [11].

flexible long rods with tapering ends. Myxospores are short, optically refractile rods or spheroids. Swarm colonies quickly adsorb Congo red and turn purple red [13].

The Myxobacteria Bank at Hoseo University possesses approximately 2,600 myxobacterial strains that were isolated in Korea. In this study, we attempted to identify tubulysin-producing strains among the myxobacterial strains in the Myxobacteria Bank by screening strains carrying tubulysin biosynthetic genes.

\section{Materials and Methods}

\section{Strains, culture conditions, and chemicals}

All myxobacterial strains used in this study were isolated from Korean soil by the Myxobacteria Bank at Hoseo University [14, 15]. Among the strains used for this study, another strain number of KYC2615 was MEHO 001, and the accession number for this strain provided by the International Depositary Authority was KCTC 13929BP. Another strain number of KYC4066 was MEHO 003, and the accession number was KCTC 14258BP. VY/3 medium [16] was used for the vegetative growth of the strains, and CYS medium [16] was used for the preparation of culture extracts. All strains were cultured at $32^{\circ} \mathrm{C}$. Synthetic tubulysin (TAM1446) was purchased from Tub Pharmaceuticals GmbH, Austria.

\section{Polymerase chain reaction (PCR)}

Two oligonucleotides，5'-GTGCAGAAYCTGCGGCTCT T-3' (H403) and 5'-AGCTGCTCCTGGAACCACA-3' (H404) were used to detect tubulysin bioisynthetic genes using PCR. Two oligonucleotides, 5'-GAGTTTGATCCTGGCTCAG-3' (27f) and 5'-AGAAAGGAGGTGATCCAGCC-3' (1525r) that were reported previously [17] were used for PCR amplification of 16S rRNA genes. PCR was performed using 2X Taq PCR Pre-Mix (Solgent, Korea). For the PCR analysis, 35 cycles that included denaturation at $94^{\circ} \mathrm{C}$ for $40 \mathrm{sec}$, annealing at $60^{\circ} \mathrm{C}$ for $20 \mathrm{sec}$, and extension at $72^{\circ} \mathrm{C}$ for $90 \mathrm{sec}$ were performed. The PCR products were analyzed by agarose gel electrophoresis.

DNA sequences of the PCR products were determined by Macrogen (Korea) and were analyzed using the BLAST program [18]. The phylogenetic analysis of the strains based on 16S rRNA sequences was performed using the MEGA-X program [19] and the 16S rRNA database from EZBioCloud [20].

\section{Preparation of culture extracts}

Myxobacterial strains were cultured in CYS liquid media with 1\% Amberlite XAD-16 resin (Sigma, USA) for 7 days. The resin was harvested and extracted using methanol. Methanol was evaporated from the extract, and the residue was dissolved in a mixture of ethyl acetate and water in a 1:1 ratio (v/v). After the ethyl acetate and water layers were separated, the ethyl acetate layer was recovered and dried. The dried residue was dissolved in methanol.

\section{High-performance liquid chromatography (HPLC)}

HPLC was performed using an HPLC system (1260 VL Infinity Series; Agilent, USA) equipped with a Zorbax SB-C18 column $(4.6 \times 150 \mathrm{~mm}, 5 \mu \mathrm{m})$ (Agilent) Mobile phase A was water and mobile phase B was acetonitrile, and both mobile phases contained $0.1 \%$ formic acid. The gradient elution at a flow rate of $0.5 \mathrm{ml} / \mathrm{min}$ was performed as follows: $0-5 \mathrm{~min} 30 \% \mathrm{~B}$ (isocratic), 5-25 min 30-60\% B (linear gradient), 25-30 min 60$100 \%$ B (linear gradient), and 30-35 min 100\% B (isocratic).

\section{LC-MS analysis}

Liquid chromatography mass spectrometry (LC-MS) was performed using an Accelar UHPLC (Thermo Scientific, USA) equipped with an Acquity UPLC ${ }^{\circledR}$ BEH C18 column $(2.1 \times 150 \mathrm{~mm}, 1.7 \mu \mathrm{m})$ and an LTQ-Orbitrap XL high-resolution mass analyzer located at Gyeonggi 
Bio-Center. Mobile phase A was water, mobile phase B was acetonitrile, and both mobile phases contained $0.1 \%$ formic acid. The gradient elution at a flow rate of $0.4 \mathrm{ml} /$ min was performed as follows: $0-1 \mathrm{~min} 20 \% \mathrm{~B}$ (isocratic), 1-20 min 20-80\% B (linear gradient), 20-24 min 80$100 \% \mathrm{~B}$ (linear gradient), and $24-27 \mathrm{~min} 100 \% \mathrm{~B}$ (isocratic).

\section{Results}

\section{Development of PCR primers for the detection of tubulysin biosynthesis genes}

In this study, we used PCR analyses in an attempt to select a tubulysin-producing strain by screening strains with tubulysin biosynthetic genes from among the 81 strains of Cystobacteraceae owned by the Myxobacteria Bank, and following selection, we confirmed the production of tubulysins. To achieve this, PCR primers that specifically detect only tubulysin biosynthesis genes are required. However, as such primers have not been previously characterized, we first endeavored to develop primers that specifically detect the tubulysin biosynthetic genes.

To develop PCR primers specific for the tubulysin biosynthesis genes, the nucleotide sequences of the tubulysin biosynthesis genes from $A$. disciformis An d48 and Cystobacter sp. SBCb004 (GenBank accession numbers: AJ620477, GU002154) were aligned. Conserved sequence regions in both strains were selected and used to design 7 pairs of PCR primers (Fig. 1, Table S1). These primer pairs were first used for PCR amplification on 10 strains that were randomly selected to test if they specifically amplified the tubulysin biosynthetic genes. The analysis of PCR products by agarose gel electrophoresis revealed that excessive amounts of nonspecific bands were generated from the six pairs of primers, and thus, strains with and without tubulysin biosynthetic genes could not be distinguished. However, the H403/ H404 primer pair specifically amplified the end of the $t u b B$ gene and the beginning of the $t u b C$ gene. Therefore, the H403 and H404 primer pairs were selected as primers for detecting the tubulysin biosynthetic genes.

\section{Screening of strains carrying tubulysin biosynthesis genes by PCR}

When PCR was performed on 81 strains using the
H403 and H404 primer pair, it was found that a PCR product of approximately $1.4 \mathrm{~kb}$ in size was generated from 8 strains (KYC1032, KYC2615, KYC2714, KYC2827, KYC2836, KYC2837, KYC2840, and KYC4066). Analysis of the nucleotide sequences of the resulting PCR products indicated that all of the PCR products possessed the end of the $t u b B$ gene and the beginning portion of the $t u b C$ gene, and these products exhibited greater than 83\% similarity to those from Cystobacter sp. SBCb004. For example, the sequence of the PCR product (1,399 bp) obtained from the KYC4066 strain was 85\% identical to that of the tubB-tubC gene site of Cystobacter sp. SBCb004 (Fig. S1) This confirmed that the H403 and H404 primer pair specifically amplified the tubulysin biosynthesis genes.

\section{Phylogenetic analysis of the strains}

Six of the 8 selected strains (KYC1032, KYC2615 (= MEHO 001), KYC2714, KYC2836, KYC2837, and KYC4066 (= MEHO 003)) were pure isolated strains, while KYC2827 and KYC2840 were not. The 27f and $1525 \mathrm{r}$ primers were used to amplify the $16 \mathrm{~S}$ rRNA gene of 6 pure isolated strains by PCR. The nucleotide sequences of the resulting PCR products were determined and then used to identify the strains according to phylogenetic analysis. The nucleotide sequences of the 16S rRNA genes from the KYC2615 and KYC4066 strains were $99.11 \%$ and $99.79 \%$ identical, respectively, to that of DSM $2261^{\mathrm{T}}$, which is the type strain of Archangium gephyra (Fig. 2). The KYC1032 and KYC2714 strains possessed 16S rRNA gene sequences that were $99.52 \%$ and $99.93 \%$ identical, respectively, to those of ATCC $25190^{\mathrm{T}}$, ATCC $25191^{\mathrm{T}}$, and DSM $14722^{\mathrm{T}}$, the type strains of Stigmatella (Fig. 2). The nucleotide sequences of the 16S rRNA genes of the KYC2836 and KYC2837 strains were $99.31 \%$ and $99.83 \%$ identical, respectively, to that of $\mathrm{MCy} 10943^{\mathrm{T}}$, the type strain of Vitosangium cumulatum (Fig. 2). Therefore, among the six strains, two strains appeared to be A. gephyra, another two strains were Stigmatella sp., and the remaining two strains were $V$. cumulatum.

\section{Analysis of culture extracts}

To test if the 8 strains selected through PCR detection actually produce tubulysins, culture extracts were prepared and analyzed by HPLC. When the culture extract 


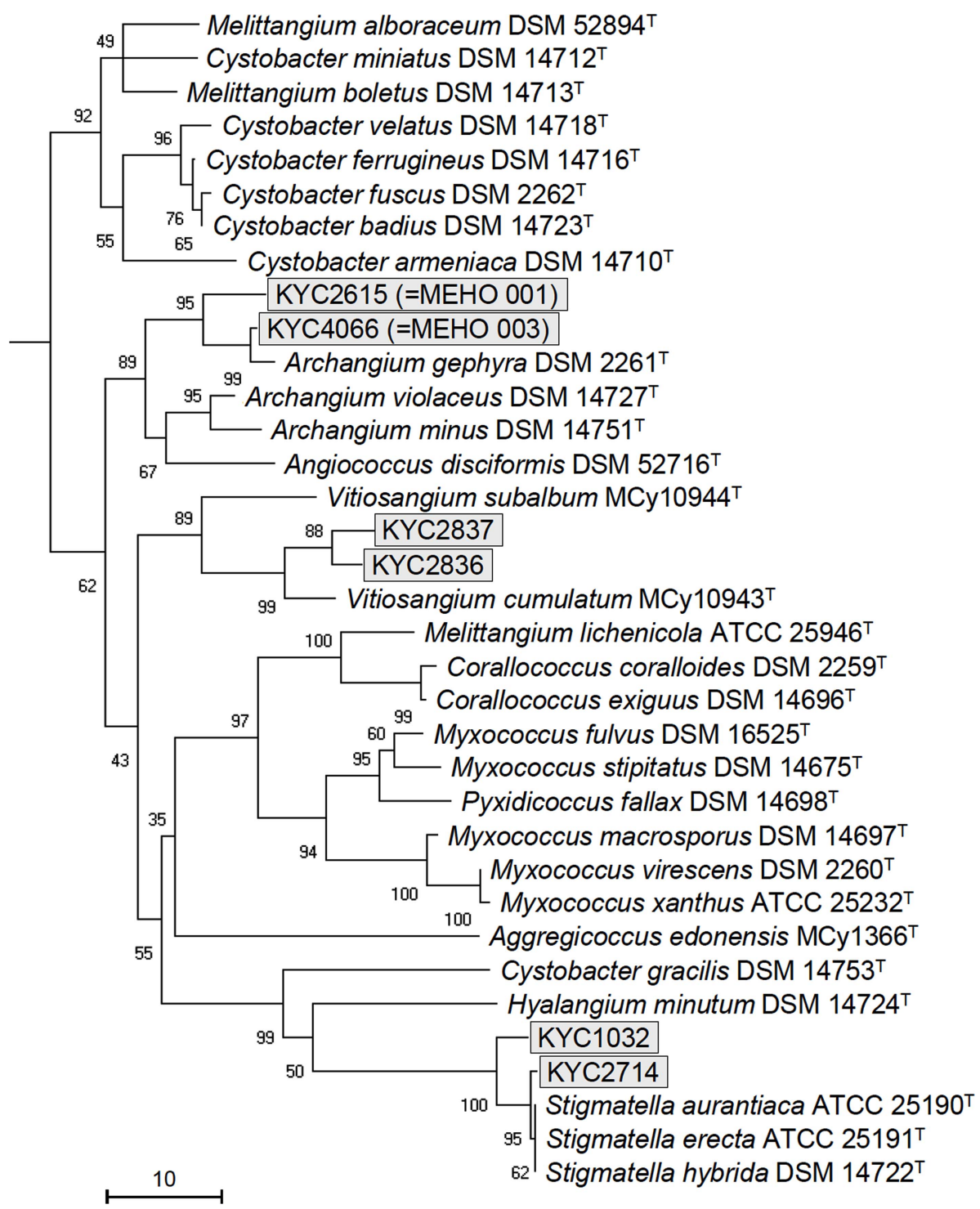

Fig. 2. Phylogenetic dendrogram of the strains based on 165 rRNA sequences. The phylogenetic dendrogram was generated using the Neighbor-Joining method [23]. The bootstrap values are expressed as percentages of 1,000 replications. Anaeromyxobacter dehalogenans $2 \mathrm{CP}-1^{\top}$ (AF382396) was used as an out-group (not shown). Bar, 10 substitutions in 1,452 nucleotide positions.

of the KYC4066 strain was analyzed, the peak substances detected at 22.58 and 25.21 min exhibited a PDA spectrum that was highly similar to that of TAM1446, a synthetic tubulysin (Fig. 3). Subsequently, these peak substances were separated by HPLC and then analyzed by mass spectrometry. The $22.58 \mathrm{~min}$ peak substance exhibited an $\mathrm{m} / \mathrm{z}$ value of $830.4334\left([\mathrm{M}+\mathrm{H}]^{+}\right)$that was highly similar to the theoretical molecular weight of tubulysin B $\left(830.4368\left([\mathrm{M}+\mathrm{H}]^{+}\right)\right)$, and the $25.21 \mathrm{~min}$ peak substance possessed a $\mathrm{m} / \mathrm{z}$ value of 844.4487 $\left([\mathrm{M}+\mathrm{H}]^{+}\right)$that was highly similar to the theoretical molecular weight of tubulysin A $\left(844.4525\left([\mathrm{M}+\mathrm{H}]^{+}\right)\right)$ (Fig. 4). When all the possible chemical formulas possessing a molecular weight within Delta $(\mathrm{ppm}) \pm 5$ were analyzed, no other substances produced by myxobacteria other than tubulysin A and B were found. Therefore, the $22.58 \mathrm{~min}$ and $25.21 \mathrm{~min}$ peak substances detected in the culture extract from the KYC4066 strain were highly 


\section{A. HPLC chromatogram}

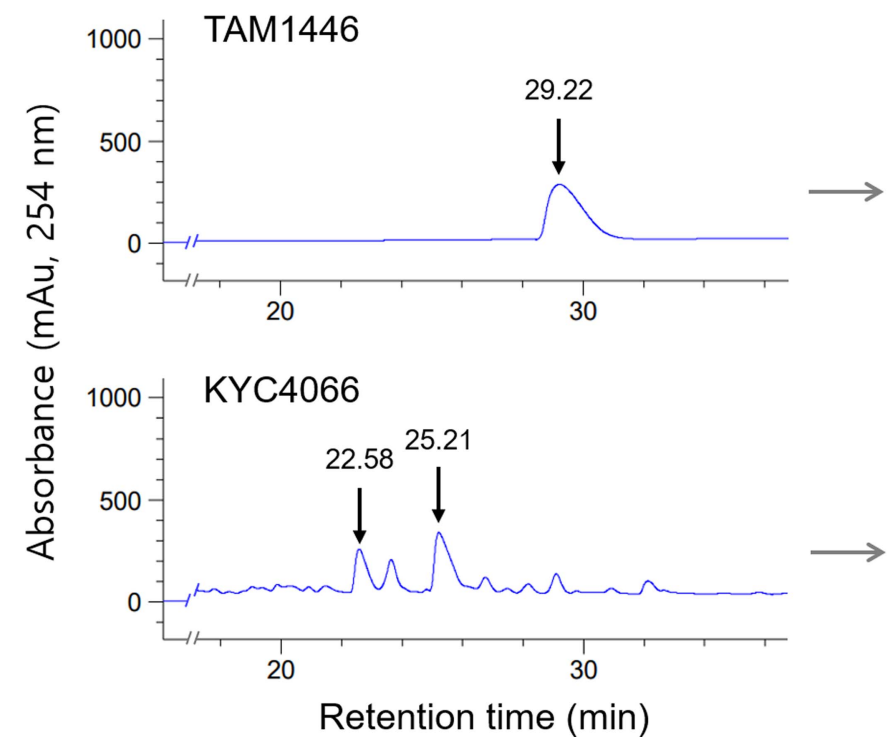

B. PDA spectrum
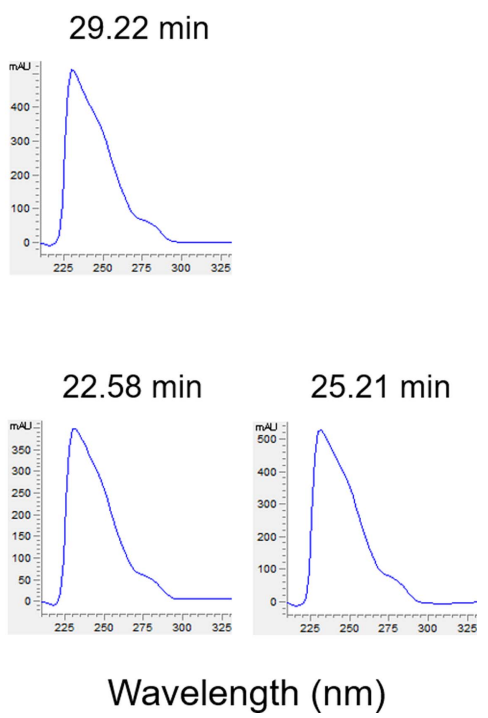

Fig. 3. HPLC chromatogram of the culture extract from Archangium gephyra KYC4066 and PDA spectrums of HPLC peaks. The culture extract from A. gephyra KYC4066 was separated using an HPLC method, and the PDA spectrum of the peaks was compared to that of TAM1446, a synthetic tubulysin.

likely to be tubulysin B and tubulysin A, respectively.

\section{Discussion}

Searching for microorganisms that produce specific substances requires a great deal of time and effort. Even more efforts are required when the microorganisms that produce a specific target substance are not common or when the substance is very difficult to analyze due to small production amounts. Tubulysins inhibit the cytoskeleton and exhibit excellent anticancer activity. Therefore, many studies examining this substance have been conducted. However, only a few of the strains producing trubulysins are known worldwide. Additionally, the trubulysin producing strains produce trace amounts of tubulsysins. In this study, we developed a primer pair that specifically detects the tubulysin biosynthesis genes, and used it in PCR analyses to select 8 strains containing the tubulysin biosynthesis gene. By analyzing the culture extracts of these strains, we confirmed that A. gephyra KYC4066 produced substances that were presumed to be tubulysin A and B. Selection of microbial strains by this method is expected to be useful in the future for the selection of strains that produce var- ious other substances.

Of the 81 strains used in this study, 24 were presumed to be A. gephyra. Of these, only two strains, KYC2615 and KYC4066, were detected to possess the tubulysin biosynthesis gene, while most of the other $A$. gephyra strains were detected not to have the tubulysin biosynthesis gene. We also could not locate the tubulysin biosynthesis genes in the genome of DSM $2261^{\mathrm{T}}$, the type strain of A. gephyra (GenBank accession number: CP011509). Some secondary metabolic biosynthesis genes of myxobacteria, including the carotenoid biosynthesis genes, are commonly found in almost all myxobacterial strains [3]. However, some genes were found only in specific strains. For example, chivosazol and etnangien biosynthetic genes are found in the So ce56 strain of Sorangium cellulosum; however, they were not found in the So0157-2 strain belonging to the same species [21, 22]. It appears that tubulysin is another example in which only a few strains possess a certain biosynthetic gene, while most of the other strains do not despite belonging to the same species.

Tubulysins were first reported to be produced by the myxobacteria A. disciformis and A. gephyra [6], and it was later reported to be produced by Cystobacter sp. [11]. 

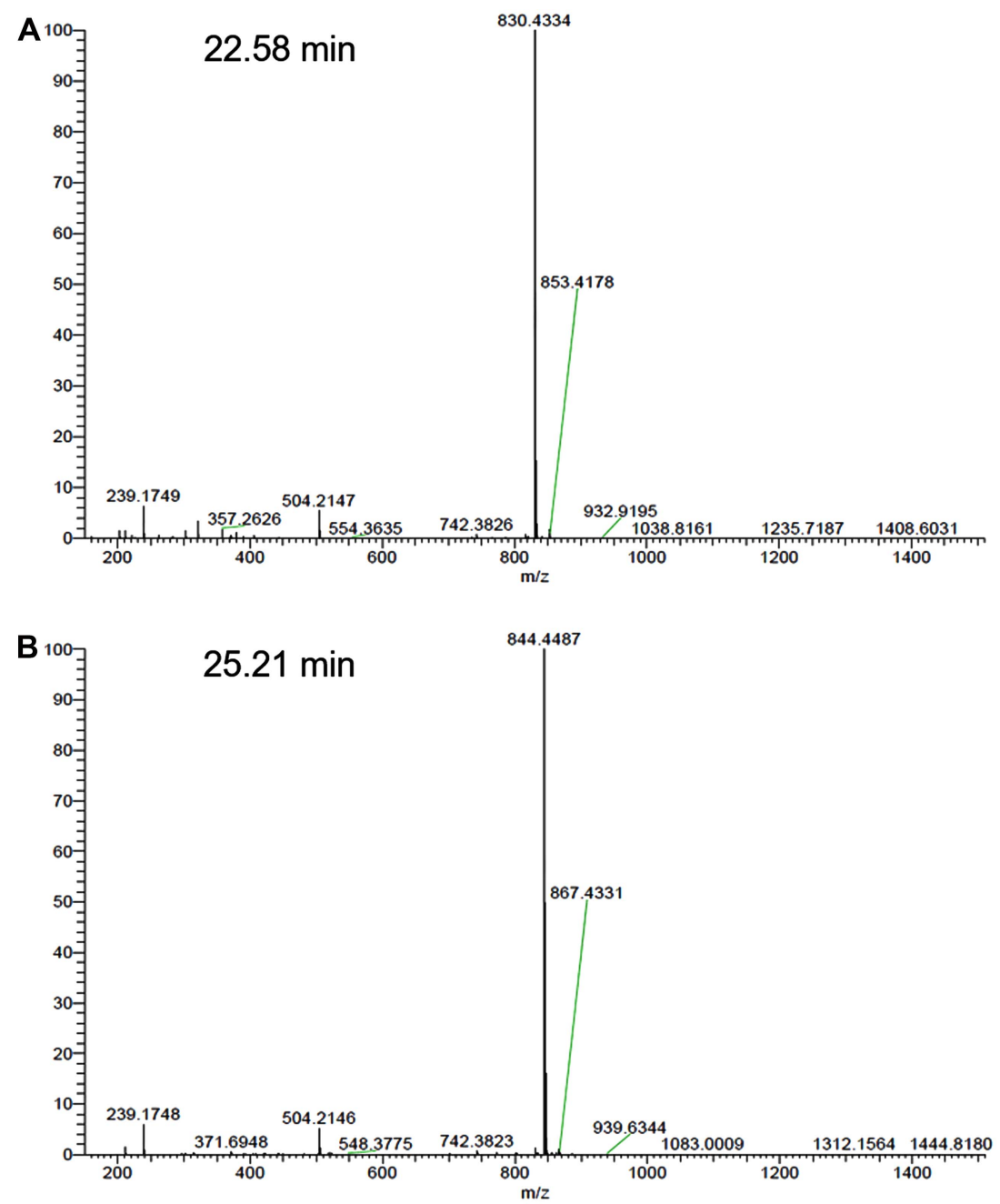

Fig. 4. Mass spectrums of the HPLC peak substances produced by A. gephyra KYC4066.

Sequences similar to those of the tubulysin biosynthetic genes were also found in the genome of a strain belonging to the genus Vitiosangium (GenBank accession number: NZ_PZOX01000000), and some gene fragments were found in the genome of $S$. aurantiaca [11]. In this study, it was determined that two strains of $A$. gephyra, two strains of Stigmatella, two strains of V. cumulatum, and two strains of unidentified myxobacteria possessed tubulysin biosynthesis genes. As tubulysins are produced in a very small amount while the culture extracts contain many other substances, it was difficult to determine if the strains produced tubulysins by the simple HPLC analysis of the culture extract. Therefore, it remains unknown if all these strains produce tubulysins. It is also unknown if all these strains possess a complete set of tubulysin biosynthetic genes. However, we found that KYC4066, one of the selected strains, produced putative tubulysin A and B. Thus, we expected that it is highly likely that these strains produce tubulysins.

\section{Conflict of Interest}

The authors have no financial conflicts of interest to declare.

\section{References}

1. Gerth K, Pradella S, Perlova O, Beyer S, Müller R. 2003. Myxobacteria: proficient producers of novel natural products with various biological activities - past and future biotechnological aspects with the focus on the genus Sorangium.J. Biotechol. 106: 233-253. 
2. Schaberle TF, Lohr F, Schmitz A, Konig GM. 2014. Antibiotics from myxobacteria. Nat. Prod. Rep. 31: 953-972.

3. Hyun H, Cho K. 2018. Secondary metabolites of myxobacteria. Korean J. Microbiol. 54: 175-187.

4. Gerth K, Bedorf N, Höfle G, Irschik H, Reichenbach H. 1996. Epothilons $A$ and $B$ : antifungal and cytotoxic compounds from Sorangium cellulosum (Myxobacteria). Production, physicochemical and biological properties. J. Antibiot. 49: 560-563.

5. Stein A. 2010. Ixabepilone. Clin. J. Oncol. Nurs. 14: 65-71.

6. Sasse F, Steinmetz H, Heil J, Höfle G, Reichenbach H. 2000. Tubulysins, new cytostatic peptides from myxobacteria acting on microtubuli. Production, isolation, physico-chemical and biological properties. J. Antibiot. 53: 879-885.

7. Murray BC, Peterson MT, Fecik RA. 2015. Chemistry and biology of tubulysins: antimitotic tetrapeptides with activity against drug resistant cancers. Nat. Prod. Rep. 32: 654-662.

8. Reddy JA, Dorton R, Bloomfield A, Nelson M, Dircksen C, et al. 2018. Pre-clinical evaluation of EC1456, a folate-tubulysin anticancer therapeutic. Sci. Rep. 8: 8943.

9. Szigetvari NM, Dhawan D, Ramos-Vara JA, Leamon CP, Klein PJ, Ruple AA, et al. 2018. Phase I/II clinical trial of the targeted chemotherapeutic drug, folate-tubulysin, in dogs with naturallyoccurring invasive urothelial carcinoma. Oncotarget 9: 3704237053.

10. Steinmetz H, Glaser N, Herdtweck E, Sasse F, Reichenbach $H$, Höfle G. 2004. Isolation, crystal and solution structure determination, and biosynthesis of tubulysins - powerful inhibitors of tubulin polymerization from myxobacteria. Angew. Chem. Int. Ed. 43: 4888-4892.

11. Chai Y, Pistorius D, Ullrich A, Weissman KJ, Kazmaier U, Müller R. 2010. Discovery of 23 natural tubulysins from Angiococcus disciformis An d48 and Cystobacter SBCb004. Chem. Biol. 17: 296-309.

12. Sandmann A, Sasse F, Müller R. 2004. Identification and analysis of the core biosynthetic machinery of tubulysin, a potent cytotoxin with potential anticancer activity. Chem. Biol. 11: 10711079.

13. Reichenbach H. 2005. Myxococcales, pp. 1059-1144. In Brenner
DJ, Krieg NR, Staley JT, Garrity GM. (eds.), Bergey's manual of systematic bacteriology, 2nd Ed. Bergey's Manual Trust, East Lansing, MI, USA.

14. Park S, Lee B, Kim J, Lee C, Jang E, Cho K. 2004. Isolation and characterization of bacteriolytic wild myxobacteria. Korean J. Microbiol. Biotechnol. 32: 218-223.

15. Hyun H, Chung J, Lee H, Youn J, Lee C, Kim D, et al. 2009. Isolation of cellulose-degrading myxobacteria Sorangium cellulosum. Korean J. Microbiol. 45: 48-53.

16. Shin H, Youn J, An D, Cho K. 2013. Production of antimicrobial substances by strains of myxobacteria Corallococcus and Myxococcus. Korean J. Microbiol. Biotechnol. 41: 44-51.

17. Rainey FA, Ward-Rainey N, Kroppenstedt RM, Stackebrandt E. 1996. The genus Nocardiopsis represents a phylogenetically coherent taxon and a distinct actinomycete lineage: proposal of Nocardiopsaceae fam. nov. Int. J. Syst. Bacteriol. 46: 1088-1092.

18. Johnson M, Zaretskaya I, Raytselis Y, Mereshuk Y, McGinnis S, Madden TL. 2008. NCBI BLAST: a better web interface. Nucleic Acids Res. 36: W5-W9.

19. Tamura K, Stecher G, Peterson D, Filipski A, Kumar S. 2013. MEGA6: Molecular evolutionary genetics analysis version 6.0. Mol. Biol. Evol. 30: 2725-2729.

20. Yoon SH, Ha SM, Kwon S, Lim J, Kim Y, Seo H, et al. 2017. Introducing EzBioCloud: A taxonomically united database of $16 \mathrm{~S}$ rRNA and whole genome assemblies. Int. J. Syst. Evol. Microbiol. 67: 1613-1617.

21. Pradella S, Hans A, Spröer C, Reichenbach H, Gerth K, Beyer S. 2002. Characterisation, genome size and genetic manipulation of the myxobacterium Sorangium cellulosum So ce56. Arch. Microbiol. 178: 484-492.

22. Han K, Li ZF, Peng R, Zhu LP, Zhou T, Wang LG, et al. 2013. Extraordinary expansion of a Sorangium cellulosum genome from an alkaline milieu. Sci. Rep. 3: 2101.

23. Saitou N, Nei M. 1987. The neighbor-joining method: a new method for reconstructing phylogenetic trees. Mol. Biol. Evol. 4: 406-425. 